\title{
Engineering spin propagation across a hybrid organic/inorganic interface using a polar layer
}

\author{
L. Schulz ${ }^{1}$, L. Nuccio ${ }^{2}$, M. Willis ${ }^{2}$, P. Desai ${ }^{2}$, P. Shakya ${ }^{2}$, T. Kreouzis ${ }^{2}$, V. K. Malik ${ }^{1}$, C. Bernhard ${ }^{1}$, \\ F. L. Pratt ${ }^{3}$, N. A. Morley ${ }^{4}$, A. Suter ${ }^{5}$, G. J. Nieuwenhuys ${ }^{5}$, T. Prokscha ${ }^{5}$, E. Morenzoni ${ }^{5}$, W. P. Gillin ${ }^{2 \star}$ \\ and A. J. Drew ${ }^{1,2 \star}$
}

\begin{abstract}
Spintronics has shown a remarkable and rapid development, for example from the initial discovery of giant magnetoresistance in spin valves ${ }^{1}$ to their ubiquity in hard-disk read heads in a relatively short time. However, the ability to fully harness electron spin as another degree of freedom in semiconductor devices has been slower to take off. One future avenue that may expand the spintronic technology base is to take advantage of the flexibility intrinsic to organic semiconductors (OSCs), where it is possible to engineer and control their electronic properties and tailor them to obtain new device concepts ${ }^{2}$. Here we show that we can control the spin polarization of extracted charge carriers from an OSC by the inclusion of a thin interfacial layer of polar material. The electric dipole moment brought about by this layer shifts the OSC highest occupied molecular orbital with respect to the Fermi energy of the ferromagnetic contact. This approach allows us full control of the spin band appropriate for charge-carrier extraction, opening up new spintronic device concepts for future exploitation.
\end{abstract}

The development and understanding of new hybrid organic/inorganic interfaces will enable considerable progress in organic spintronics for technological purposes, including processing elements, sensors, memories and conceptually different future applications. In addition to the 'standard' spintronic applications, newly developed interfaces could bring spintronic effects to the field of organic light-emitting diodes (OLEDs), as well as in the fast progressing field of organic field-effect transistors. For example, the injection of carriers with a controlled spin state could enable the amplification of either singlet or triplet exciton states ${ }^{2}$ leading to a significant increase in the efficiency of the electroluminescence in OLEDs. Although these considerations are conceptually straightforward, no efficiency amplification has yet been reported in the literature, despite several attempts ${ }^{3}$. The failure of those approaches was caused by the simple reason that light emission can be detected starting from an applied voltage of a few volts, whereas state-of-the-art spin injection in organic materials persists to a maximum of around $1 \mathrm{~V}$ (refs 4-6). As yet, this is unexplained. Further complications arise from the fact that various reports on working devices show a wide spread of performances for apparently similar structures, highlighting the issue of reproducibility ${ }^{7-9}$. The poor reproducibility is mainly due to the unknown interplay between processing and spin transfer performance and there is little deterministic control of the interface properties. However, it has recently been demonstrated that the insertion of a barrier layer on top of an organic material can increase reproducibility for aluminium tris (8-hydroxyquinoline) ( $\mathrm{Alq}_{3}$ )-based spin valves ${ }^{10}$.

Despite the reproducibility issues, the potential for organic spintronic devices seems enormous, with reports of very large spin-valve magnetoresistance at low temperature ${ }^{11}$. One clear goal, whatever the application, is to be able to select and control the injection and extraction of spins in organic materials. This in turn requires the exquisite control of the electronic and structural states at the hybrid organic/inorganic interface ${ }^{12}$, which until recently has been only passively determined through experiments, rather than proactively and deterministically controlled. One of the key advantages of OSCs is the ease with which their electronic nature can be altered, and one such way is the use of polar materials to tune the alignment between the electrode Fermi level and the OSC molecular levels ${ }^{13,14}$. This has recently been demonstrated by covering a $\mathrm{TiO}_{2}$ electrode with an oriented ionic molecular

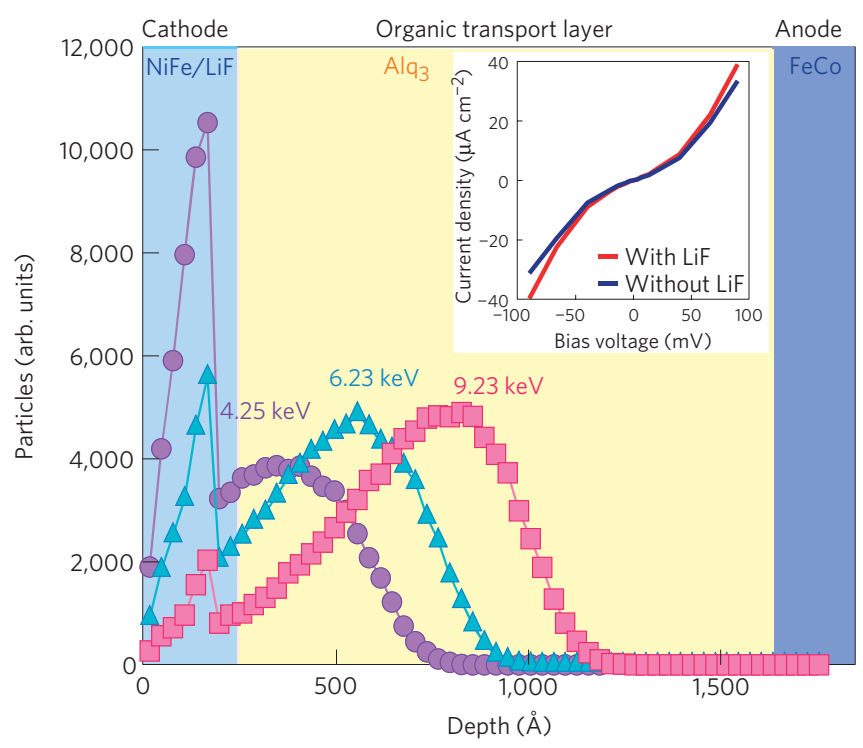

Figure 1 | A schematic of the device structure. The muon's stopping profile is plotted for sample A, calculated with a Monte Carlo algorithm (see the Methods section). A similar profile is obtained in sample B. Inset: The IV characteristics measured on the two small-area devices-with LiF and without LiF (sample A and B, respectively).

${ }^{1}$ Department of Physics and Fribourg Center for Nanomaterials, University of Fribourg, Chemin du Musée 3, CH-1700 Fribourg, Switzerland, ${ }^{2}$ Queen Mary University of London, School of Physics, Mile End Road, London E1 4NS, UK, ${ }^{3}$ ISIS Pulsed Neutron and Muon Source, Rutherford Appleton Laboratory, Chilton, Didcot OX11 OQX, UK, ${ }^{4}$ Department of Materials Science and Engineering, University of Sheffield, Mappin Street, Sheffield S1 3JD, UK, ${ }^{5}$ Laboratory for Muon-Spin Spectroscopy, Paul Scherrer Institute, CH-5232 Villigen PSI, Switzerland. *e-mail:W.Gillin@qmul.ac.uk; A.J.Drew@qmul.ac.uk. 

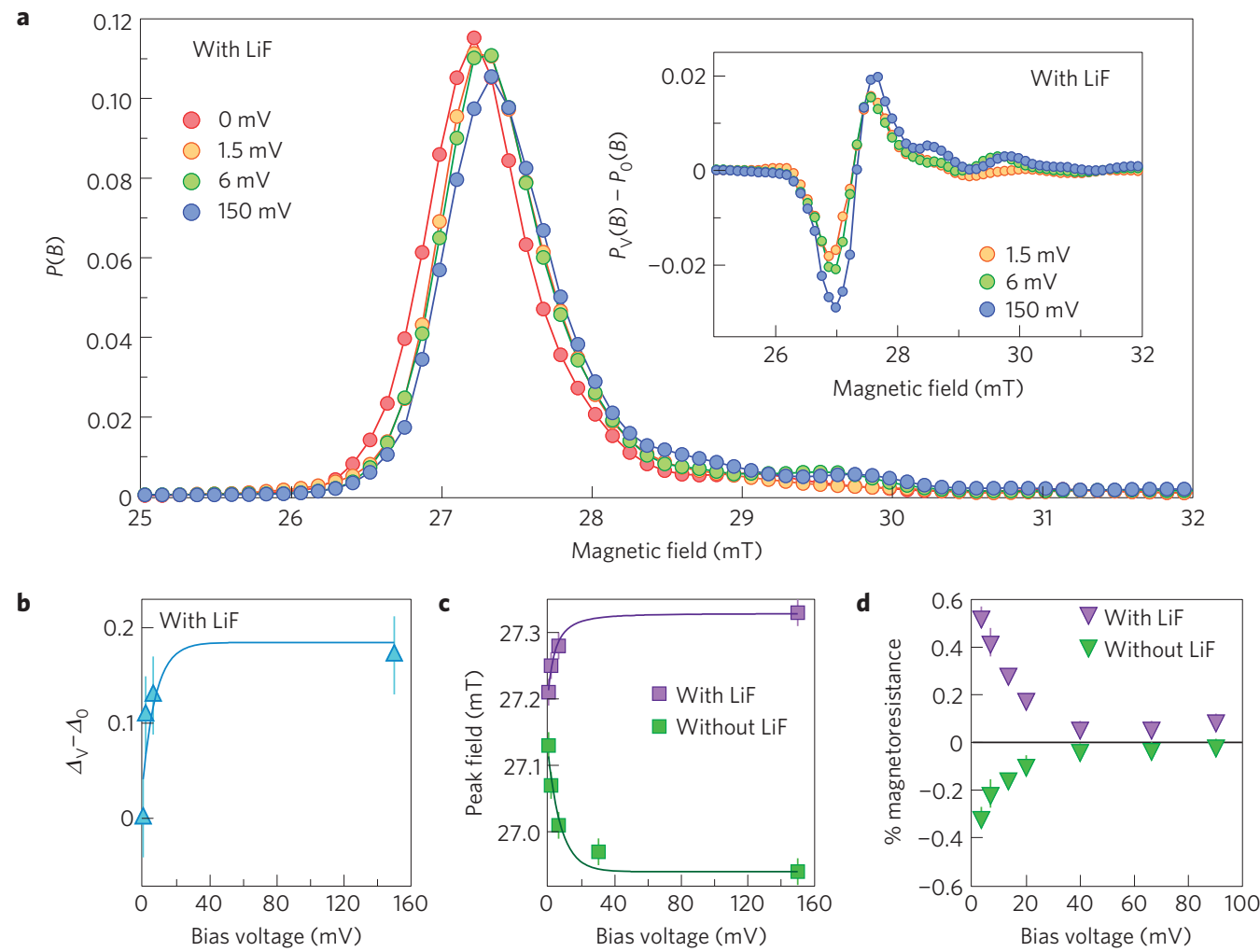

Figure 2 | Spin-polarized charge carriers are present in the OSC, close to the top NiFe interface. a, Probability distribution of magnetic field inside the device with LiF (sample A) at several different voltages. Inset: The difference between the data with and without an applied voltage, where a clear increase in the difference signal amplitude is observed at higher voltages. b,c, The change in line-shape skewness (b) and peak field (c) clearly saturates at higher voltages. A clear reversal in the voltage dependence of the peak field is observed in $\mathbf{c}$ when the LiF layer is omitted. $\mathbf{d}$, The magnetoresistance for devices with and without LiF. The reduction of magnetoresistance occurs at similar voltages to the saturation of the LE- $\mu$ SR data shown in $\mathbf{b}$ and $\mathbf{c}$. Muon measurements were taken at a temperature of $10 \mathrm{~K}$ and at an energy of $6.23 \mathrm{keV}$. The magnetoresistance was taken at $20 \mathrm{~K}$. In $\mathbf{b}$ and $\mathbf{c}$, the error bars represent one standard deviation, calculated from the Poissonian statistics of the muon data. For $\mathbf{d}$, the error bars represent an estimate of the scatter present in the magnetoresistance data. $\ln \mathbf{b}$ and $\mathbf{c}$, the lines are guides to the eye.

monolayer of amphiphilic molecules ${ }^{15}$. Unfortunately, the resultant energy level shift is not well understood for any organic/inorganic interface, especially when the electrode material is ferromagnetic ${ }^{13}$.

Here we show, using the direct spectroscopic technique lowenergy muon spin rotation ${ }^{16,17}$ (LE- $\left.\mu \mathrm{SR}\right)$, that the polar material LiF reverses the spin polarization of carriers at the NiFe interface with $\mathrm{Alq}_{3}$. LiF has the advantage that it can be vacuum-deposited over large areas, using thermal evaporation. It is a standard material used to achieve a vacuum level shift of up to $1 \mathrm{eV}$ in OLED devices ${ }^{18,19}$, via the electric dipole moment that develops as a result of the termination of the polar material at the interfaces. In our LE- $\mu$ SR experiment, two devices were measured with an active area of $16 \times 16 \mathrm{~mm}$, comprising $\mathrm{FeCo} 17 \mathrm{~nm} / \mathrm{Alq}_{3} 150 \mathrm{~nm} / \mathrm{LiF} \quad 1 \mathrm{~nm} / \mathrm{NiFe} 20 \mathrm{~nm}$ (sample A) and FeCo $17 \mathrm{~nm} / \mathrm{Alq}_{3} 150 \mathrm{~nm} / \mathrm{NiFe} 20 \mathrm{~nm}$ (sample B). Two identical devices were grown for the electrical and magnetoresistance measurements with an active area of $2 \times 2 \mathrm{~mm}$. All samples were grown sequentially, using the same conditions in the same deposition system; further details can be found in the Methods section. A schematic of the device structure of sample A is shown in Fig. 1, which also shows the muon stopping profile for the implantation energies used in our experiments. These energies were chosen to ensure that most muons stop inside the organic layer. The inset of Fig. 1 shows the current-voltage characteristics of the two smaller-area devices.

Figure $2 \mathrm{a}$ shows the distribution of local magnetization, $P(B)$, in sample $A$ at $T=10 \mathrm{~K}$ for four bias voltages of $0,1.5,6$ and $150 \mathrm{mV}$, obtained from our LE- $\mu$ SR experiments. On applying a spin-polarized current through the device, small but significant changes in the distribution $P(B)$ are observed. These changes due to the spin-polarized current can more easily be observed by taking the difference of 'voltage on' and 'voltage off' $P_{\mathrm{V}}(B)-P_{0}(B)$. This is shown in the inset of Fig. $2 \mathrm{a}$, where it can be seen that the magnetization in the sample increases as a higher spinpolarized current is passed. A quantitative description of the voltage dependence of the changes in the muon line shapes shown in Fig. 2a can be obtained by fitting the muon's time-dependent asymmetry to a skewed Lorentzian relaxation function ${ }^{16}$, comprising a skewness parameter and peak field (corresponding to the mode of the field distribution). It has previously been shown that the skewness parameter, $\Delta$, is a very sensitive probe of the polarization of the injected charge carriers ${ }^{16}$. To understand how a change in injected spin polarization alters $\Delta$, we must first define spin-majority holes or electrons to be those that extract to or inject from the spinmajority band of the relevant ferromagnetic contact, and vice versa for the spin-minority carriers. If $\Delta$ increases on the application of a spin-polarized current, the muons are measuring a higher magnetic field due to the magnetization resulting from a population imbalance of the two spin channels in favour of spin-majority electrons, either by extraction of spin-majority holes in the highest occupied molecular orbital (HOMO) or injection of spin-majority electrons in the lowest unoccupied molecular orbital (LUMO). On the other hand, if $\Delta$ decreases, the muons measure a lower magnetic field from the imbalance in favour of spin-minority carriers.

The obtained peak field and skewness, from the time-domain fits, are then plotted as a function of voltage in Fig. 2b,c. The peak field that the muons experience increases as the bias voltage 
a
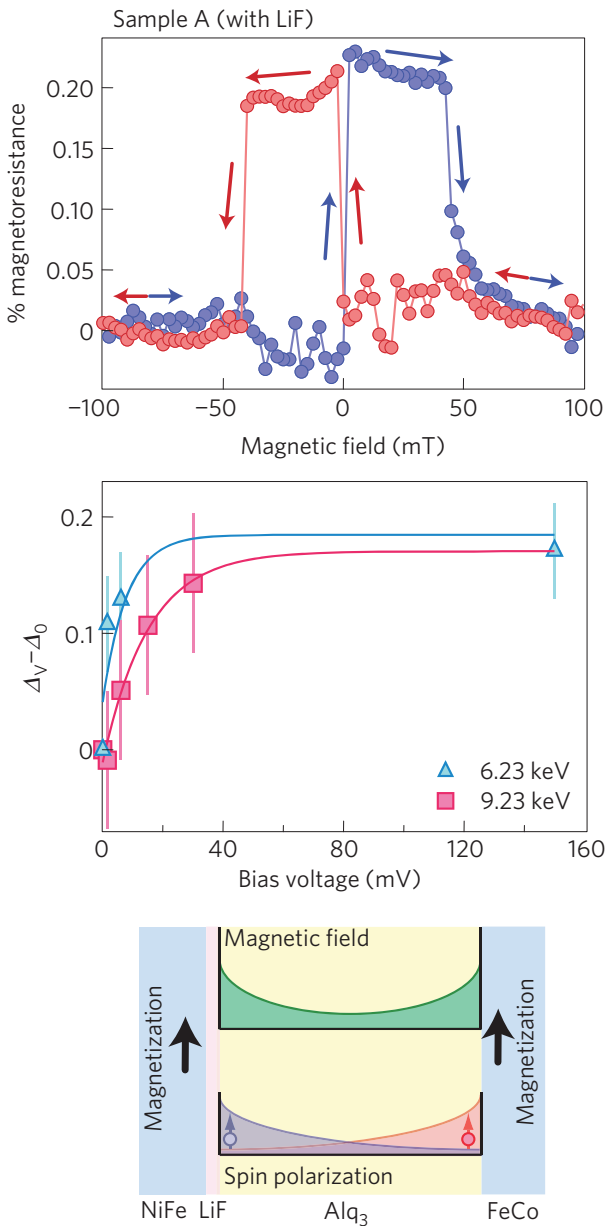

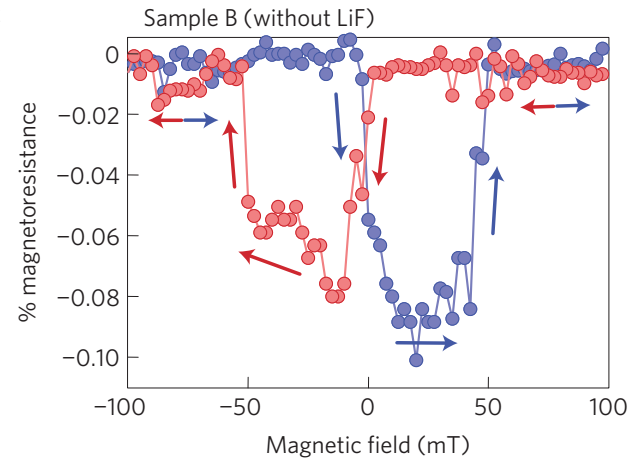

d

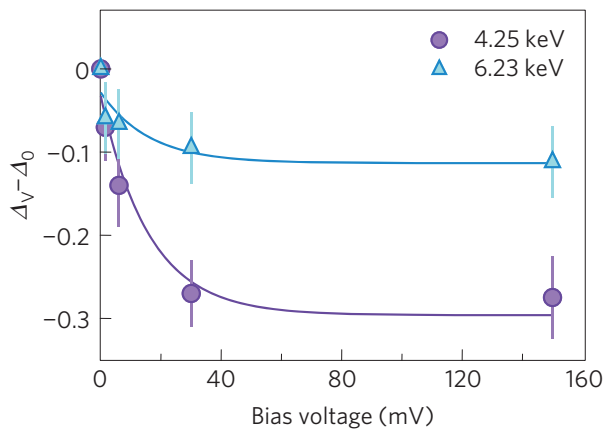

$\mathbf{f}$

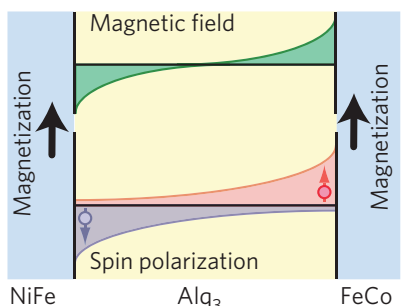

Figure 3 | A comparison of the device magnetoresistance and spin polarization close to the top interface. a, The magnetoresistance (a) and the change in $\mu$ SR line-shape skewness (b) for sample A, with the LiF layer. c,d, The magnetoresistance (c) and the change in $\mu$ SR line-shape skewness (d) for sample B, without the LiF layer. Muon measurements were taken at a temperature of $10 \mathrm{~K}$, with the magnetoresistance measurements taken at a bias of $20 \mathrm{mV}$ and at $20 \mathrm{~K}$. As can be seen from a to $\mathbf{d}$, there is a clear reversal of spin polarization as a result of the presence of the LiF layer. This is due to a change of extracted spin polarization brought about by a vacuum level shift due to the electric dipole moment induced by the LiF. e, For spin-majority hole extraction there is an increase in magnetization close to the interface. f, For spin-minority hole extraction, there is a decrease in magnetization close to the interface. In e and $\mathbf{f}$, the red shaded area and spins correspond to hole injection, whereas the blue corresponds to hole extraction. In $\mathbf{b}$ and $\mathbf{d}$, the error bars represent one standard deviation and the lines are guides to the eye. In $\mathbf{a}$ and $\mathbf{c}$, the blue and red points correspond to different magnetic field sweep directions, defined by the coloured arrows.

on the device increases and there is a noticeable enhancement of the skewness of the line shape. All observed effects here are consistent with spin-polarized charge carriers being injected into the organic layer, with this sample showing very similar behaviour to one previously studied, which had a very similar structure also including a LiF interfacial layer ${ }^{16}$. However, as is evident from all of the data presented in Fig. 2, the magnetization resulting from the spin-polarized current seems to saturate at higher voltages. It is interesting to note that spin injection into organic materials has so far been demonstrated only for voltages below approximately $1 \mathrm{~V}$, with the largest magnetoresistance observed typically at $100 \mathrm{mV}$ and below ${ }^{4,20,21}$. Indeed, as can be seen from Fig. 2 d, this very trend is observed in our devices, with the saturation voltage observed in our muon experiments corresponding well to the loss of magnetoresistance. Clearly, there is a loss of spin polarization with increasing voltage, as the $\mu$ SR line-shape skewness and peak field should scale with current if the polarization remains unchanged, whereas the magnetoresistance as plotted in Fig. 2d should scale with polarization and be independent of current.

Strong electrical dipoles are present at many OSC/metal interfaces and these interfacial dipoles can significantly alter the non-interacting equilibrium energy levels ${ }^{11,13,14,22-28}$. Thus far, little is experimentally known about the role of these vacuum level shifts on spin injection and extraction. Clearly, a spectroscopic study of spin propagation investigating the effect of such an energy shift is crucial for the understanding of the spin transport properties of hybrid ferromagnetic/organic devices. For this reason we carried out LE- $\mu$ SR measurements on a second samplenominally identical to the first one, other than the absence of the thin LiF layer at the cathode interface. Plotted in Fig. 3a-d is the bias-voltage dependence of the change in skewness for both samples and the corresponding magnetoresistance. Also plotted in Fig. 3c and $\mathrm{d}$ are the voltage-dependent peak field and magnetoresistance, respectively, for the sample without the LiF layer. It is immediately clear that the presence of the LiF layer reverses the spin polarization in the $\mathrm{Alq}_{3}$. As this phenomenon is probably due to a vacuum level shift at the interface changing the relevant spin band, we must first understand which molecular orbitals are responsible for the current in our devices before we can discuss the origin of the spin reversal. From the $I V$ characteristics (inset of Fig. 1), it can be seen that the contacts are almost ohmic with only a very small and symmetrical built-in potential. Given that the devices were being operated with 


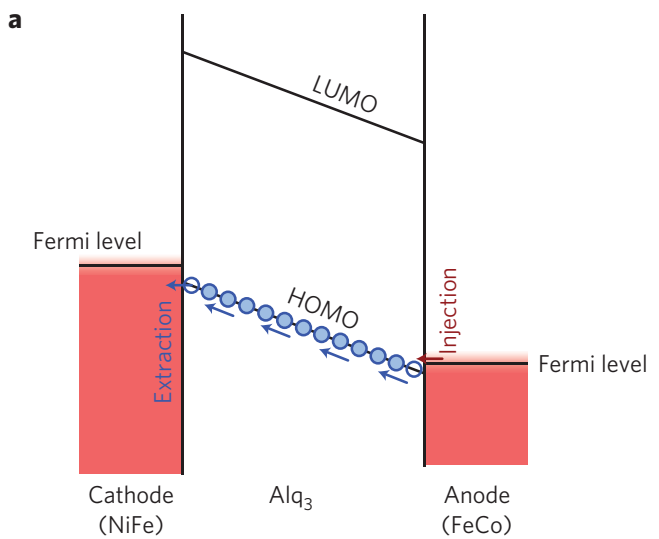

b

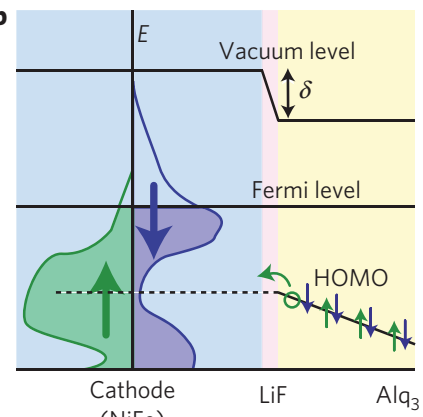

(NiFe)

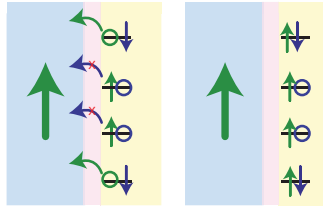

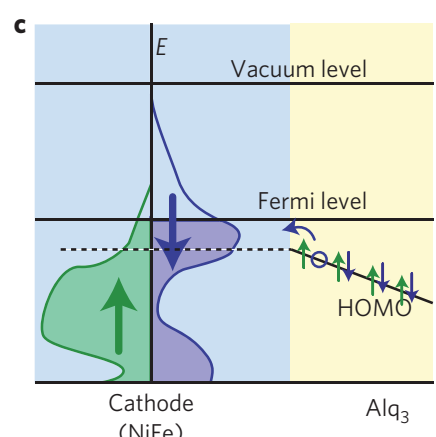

e

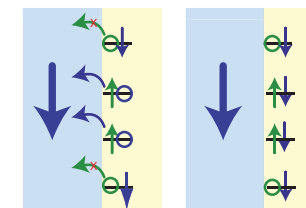

Figure 4 | Schematic of hole transport in the OSC and how a vacuum level shift leads to a change of extracted spin polarization. a, Hole injection occurs close to the Fermi level, as there must be vacant states in the ferromagnet for injection to occur. Conversely, hole extraction can take place anywhere below the Fermi level. b,d, As holes are extracted, the probability of one particular spin state dominating the extraction is related to the spin DOS at the extraction energy. For the case of the device with LiF where there is a vacuum level shift $\delta$, this results in spin-majority electron accumulation, as spin-majority holes are extracted more efficiently. c,e, For the device without the LiF layer, the vacuum level shift is not present. This results in a probability of extraction such that the most probable extracted hole polarization is spin minority, leading to an accumulation of spin-minority electrons close to the interface.

the FeCo contact as the anode, the current in the device is due to one of the two following phenomena. Either holes are being transported in the $\mathrm{HOMO}$, entering the $\mathrm{Alq}_{3}$ from the anode and exiting at the cathode, or electrons are being transported in the LUMO, entering at the cathode and exiting at the anode. As the workfunctions of the transition metals are very high $(\sim 4.5 \mathrm{eV}$ for $\mathrm{Fe}$ and $\sim 5 \mathrm{eV}$ for $\mathrm{Co}$ and $\mathrm{Ni}$; ref. 29), the possibility of electron injection into the $\mathrm{Alq}_{3}$ is unlikely, particularly given that the devices operate at less than $100 \mathrm{mV}$. It seems likely that the current in the device is therefore carried predominantly by holes traversing the HOMO (ref. 30).

Injection into an OSC can occur only from within a few $k_{\mathrm{B}} T$ of the Fermi energy of the electrode; in the case of holes, as in our devices, there need to be unoccupied states in the FeCo anode. The spin polarization of the injected holes is therefore determined by the spin density of states (DOS) at the Fermi energy of the ferromagnet. In contrast, any energy below the Fermi energy can accept a hole being extracted, provided there is a non-zero DOS

in the cathode at the energy corresponding to the HOMO of the OSC. This process is schematically shown in Fig. $4 \mathrm{a}$, where hole injection occurs near the Fermi energy and extraction below it. As stated earlier, the interface dipole introduced by LiF produces a vacuum level shift, which moves the HOMO energy relative to the Fermi level of the metal contact. This is schematically shown in Fig. 4b,c, which compares spin-dependent hole extraction with and without the LiF-induced vacuum level shift. As the spin-dependent hole extraction probability depends on the spin polarization in the cathode at the OSC HOMO energy, a shift in the HOMO would change the spin polarization of extracted holes ${ }^{13}$ (see Fig. 4). This can easily explain the data presented in Fig. 3. We would like to note that it has already been suggested that changes in coupling at the interface between the OSC and ferromagnet can alter the spin polarization of injected electrons ${ }^{11}$.

For the device without the LiF layer (Sample B, Fig. 3c,d), at the magnetic fields where the LE- $\mu$ SR measurements were carried out the magnetization of the two contacts are aligned. The change in skewness close to the cathode is negative, indicating that the total field is lowered by the spin-polarized hole extraction (upper schematic plot in Fig. 3f). This must mean that the extracted holes are spin minority, as there must be an excess of electron spins opposed to the applied field as shown in Fig. 4e, which would result in a lowering of the magnetic field observed by the muons (lower schematic plot in Fig. 3f). As the sample is in a high-resistance state when the ferromagnets have their magnetization aligned, the two electrodes must inject/extract opposite spins and therefore the anode must be injecting spin-majority holes (lower schematic plot, Fig. 3f). For the device with a LiF layer (Sample A, see Fig. 3a,b), the anode is unchanged and so should still be injecting spin-majority holes. However, if the extraction spin band is altered at the cathode, one would expect spin-majority hole extraction (see lower schematic plot, Fig. 3e). This would lead to a spin-majority electron accumulation at the cathode interface as shown in Fig. 4d, which results in an increased magnetization (see upper schematic plot, Fig. 3e) and consequently a positive change in skewness-as is observed in Fig. 3b. As both electrodes would be efficiently injecting/extracting spin-majority holes, one would expect the device to be in a low-resistance state when the two ferromagnetic layers have aligned magnetizations, as is observed in Fig. 3a. It is worth noting that changes to the tunnelling matrix element, if this is the relevant spin transfer mechanism into the OSC, could also contribute to a different spin transfer across the interface ${ }^{31,32}$. This can arise if one considers the matching condition for the k-vectors of the evanescent wave in a tunnelling barrier and the one in the ferromagnet. However, it can also lead to a bias-dependent reversal of the injected spin polarization ${ }^{31}$, which has never been observed in OSC spin-valve structures.

These results are particularly exciting for spintronics applications as they demonstrate that the dominant spin band for charge-carrier extraction can be modified through the introduction of an interfacial layer, as has already been predicted ${ }^{11,13}$. They also highlight the possibility for the engineering of more complex devices where spins can be manipulated. For example, for a metal sandwiched between two organic materials, it would be possible to extract spins with one polarization from an OSC and inject the opposite spin polarization into another OSC, and this could be switchable. If the polar layers were ferroelectrics, then it could be possible to switch the polarization of the electric dipole moment with an electric field; thus, the device could act as a spin filter or switch. Furthermore, spatially patterning alternate orientations of the polar materials may yield interference of spin-polarized currents, which could be used as a spin interferometer. Clearly, it should be possible to enhance the effects observed here, by growing epitaxial or self-assembled films with a preferred ionic orientation. It would also be interesting to carry out LE- $\mu S R$ experiments for 
both positive and negative bias, at both injection and extraction electrodes. These could shed light on the unsymmetrical nature of the voltage-dependent reduction in magnetoresistance ${ }^{5}$. It is unlikely that the polarization of extracted holes is symmetrical with positive/negative voltage as the extraction polarization depends on the DOS at the HOMO energy of the extracting electrode. The inclusion of a LiF layer should not affect the spin polarization of injected holes, because these are injected within a few $k_{\mathrm{B}} T$ of the Fermi surface. If this study was repeated with a $\mathrm{La}_{1-x} \mathrm{Sr}_{x} \mathrm{MnO}_{3}$ electrode, it would be possible to differentiate between our model and the recently suggested localized 'hot spot' model ${ }^{11}$. Finally, we would like to note that the magnitude of the magnetoresistance and muon signal is similar for both of our devices, indicating that there may be no fundamental obstacle to injecting or extracting polarized charge carriers from transition-metal ferromagnets at higher voltages. In our case, we were able to access states with different polarization through a vacuum level shift, but these may equally well be accessed by the choice of OSC or an increased bias voltage.

\section{Methods}

Samples. The devices were grown on a high-purity fused-quartz substrate with an r.m.s. roughness of less than $2 \mathrm{~nm}$. $\mathrm{Alq}_{3}$ (99.995\% pure) was purchased from Aldrich and purified by train-sublimation under a $10^{-6} \mathrm{mbar}$ vacuum. The $\mathrm{Ni}, \mathrm{Co}$ and Fe were purchased from Aldrich ( $>99.9 \%$ pure) and pressed into pellets in the proportions 80:20 Ni/Fe and 50:50 Fe/Co, which were subsequently thermally evaporated. High-purity aluminium, purchased from Aldrich, was evaporated at the edges of the sample to enable contacts to be made effectively; the contacts were well away from the centre of the beam, such that less than $2 \%$ of the beam hit the contacts $^{16}$. The deposition of the $\mathrm{Alq}_{3}$ and LiF layers was carried out using a Kurt J. Lesker SPECTROS evaporation system under $\sim 10^{-7}$ mbar. Magnetic layers were evaporated in a separate system under $\sim 10^{-6}$ mbar vacuum. A calibrated oscillating quartz-crystal monitor was used to determine the rate and thickness of all deposited layers. X-ray reflectivity was used to estimate the thickness of each layer and the interface roughness. The deposition rate of the $\mathrm{Alq}_{3}, \mathrm{Al}$ and $\mathrm{LiF}$ was maintained at $0.2 \mathrm{~nm} \mathrm{~s}^{-1}$ and that of the magnetic contacts at around $0.1 \mathrm{~nm} \mathrm{~s}^{-1}$. Shadow masks were used to define the device geometry.

LE-uSR. Positive muons decay to a positron, a muon antineutrino and an electron neutrino. The angular emission of positrons is well characterized, with the emission direction being correlated with the muon's spin at the time of decay. Thus, by measuring the direction and the timing of a statistically significant number of decay positrons, it is possible to follow directly the evolution of the spin of the ensemble of muons as a function of time after implantation. Muons can act as passive local magnetic microprobes, by directly measuring the magnetic field distribution at the implanted site with very high sensitivity (less than $0.1 \mathrm{mT}$ ). Being able to follow the evolution of the spin with time means that the local magnetic field experienced by the muon can be determined through the measurement of the Larmor precession of the muon spin, which is obtained using two positron counters mounted on opposite sides of the sample. We used a bespoke floating power supply/volt meter that could bias the sample to a high degree of accuracy $( \pm 0.1 \mathrm{nA}$ and $\pm 0.1 \mu \mathrm{V})$ while floating the sample at $\pm 10 \mathrm{kV}$. The high voltage is necessary for controlling the muon implantation energy and thus the muon stopping distribution within the device. Electrical contacts were made using spring-loaded electrodes supported by polytetrafluoroethylene blocks and the whole assembly was mounted on a high-purity Ag-coated Al plate. The magnetic field was applied parallel to the layers and perpendicular to the muon's initial spin direction and momentum. The measurements proceeded by first applying a field of $100 \mathrm{mT}$ to ensure that the ferromagnetic layers were saturated, after which the magnetic field was reduced to $27 \mathrm{mT}$. The $\mu$ SR spectra were first obtained with the current on and then with current off.

In our LE- $\mu$ SR experiment, 200-300 nm of a weakly bound van der Waals cryosolid multilayer (solid-Ar/solid- $\mathrm{N}_{2}$ ) was deposited on the downstream side of a cold metal substrate, which moderates a fraction of an intense surface muon beam to $\sim 15 \mathrm{eV}$ (with a similar r.m.s. energy spread) while conserving the initial full polarization. The epithermal muons are extracted (by applying up to $+20 \mathrm{kV}$ to the moderator substrate), transported and focused by electrostatic elements to the sample. A trigger detector provided a muon start signal by detecting secondary electrons, released by the muons when passing through a $2 \mu \mathrm{g} \mathrm{cm}^{-2}$ carbon foil onto a microchannel plate detector. The mean implantation energies were 4.25, 6.23 and $9.23 \mathrm{keV}$, controlled by choosing the appropriate moderator, transport and sample voltages. The muon's stopping profile can be calculated using a Monte Carlo algorithm TRIM.SP (ref. 32). This is shown in relation to our devices in Fig. 1. By varying the muon's stopping profile, we were able to probe the depth profile of the induced magnetization due to injected spin-polarized charge carriers. Further information regarding the technique can be found in refs $16,17,33,34$

\section{References}

1. Baibich, M. N. et al. Giant magnetoresistance of (001)Fe/(001)Cr magnetic superlattices. Phys. Rev. Lett. 61, 2472-2475 (1988).

2. Bergenti, I. et al. Spin polarised electrodes for organic light emitting diodes. Org. Electron. 5, 309-314 (2004).

3. Salis, G., Alvarado, S. F., Tschudy, M., Brunschwiler, T. \& Allenspach, R Hysteretic electroluminescence in organic light-emitting diodes for spin injection. Phys. Rev. B 70, 085203 (2004).

4. Xiong, Z. H., Wu, D., Vardeny, Z. \& Shi, J. Giant magnetoresistance in organic spin-valves. Nature 427, 821-824 (2004).

5. Dediu, V. A., Hueso, L. E., Bergenti, I. \& Taliani, C. Spin routes in organic semiconductors. Nature Mater. 8, 707-716 (2009).

6. Lin, R., Wang, F., Rybicki, J., Wohlgenannt, M. \& Hutchinson, K. A. Distinguishing between tunnelling and injection regimes of ferromagnet/organic semiconductor/ferromagnet junctions. Phys. Rev. B 81, 195214 (2010).

7. Jiang, S. B., Pearson, J. E. \& Bader, S. D. Absence of spin transport in the organic semiconductor Alq(3). Phys. Rev. B 77, 035303 (2008).

8. Schoonas, J. J. H. M. et al. Magnetoresistance in hybrid organic spin valves at the onset of multiple-step tunnelling. Phys. Rev. Lett. 103, 146601 (2009).

9. Sun, D. L. et al. Giant magnetoresistance in organic spin valves. Phys. Rev. Lett. 104, 236602 (2010).

10. Dediu, V. et al. Room-temperature spintronic effects in $\mathrm{Alq}_{3}$-based hybrid devices. Phys. Rev. B 78, 115203 (2008).

11. Barraud, C. et al. Unravelling the role of the interface for spin injection into organic semiconductors. Nature Phys. 6, 615-620 (2010).

12. Cinchetti, M. et al. Determination of spin injection and transport in a ferromagnet/organic semiconductor heterojunction by two-photon photoemission. Nature Mater. 8, 115-119 (2009).

13. Atodiresei, N. et al. Design of the local spin polarization at the organic-ferromagnetic interface. Phys. Rev. Lett. 105, 066601 (2010).

14. Campbell, I. H. et al. Controlling charge injection in organic electronic devices using self-assembled monolayers. Appl. Phys. Lett. 71, 3528-3530 (1997).

15. Bolink, H. J. et al. Molecular ionic junction for enhanced electronic charge transfer. Langmuir 25, 79-83 (2009).

16. Drew, A. J. et al. Direct measurement of the electronic spin diffusion length in a fully functional organic spin valve by low-energy muon spin rotation. Nature Mater. 8, 109-114 (2009).

17. Morenzoni, E. et al. Generation of very slow polarized positive muons. Phys. Rev. Lett. 72, 2793-2796 (1994).

18. Ishi, H. et al. Energy-level alignment at model interfaces of organic electroluminescent devices studied by UV photoemission: Trend in the deviation from the traditional way of estimating the interfacial electronic structures. IEEE J. Sel. Top. Quantum Electron. 2, 24-33 (1998).

19. Shii, H. \& Seki, K. Energy level alignment at organic/metal interfaces studied by UV photoemission: Breakdown of traditional assumption of a common vacuum level at the interface. IEEE Trans. Electron Devices 44, 1295-1301 (1997).

20. Wang, F. J., Yang, C. G., Vardeny, Z. \& Li, X. Spin response in organic spin valves based on $\mathrm{La}_{2 / 3} \mathrm{Sr}_{1 / 3} \mathrm{MnO}_{3}$ electrodes. Phys. Rev. B 75, 245324 (2007).

21. Wang, F. J. \& Vardeny, Z. V. Recent advances in organic spin-valve devices. Synth. Met. 160, 210-215 (2010).

22. Majumdar, S., Majumdar, H. S., Laiho, R. \& Osterbacka, R. Comparing small molecules and polymer for future organic spin-valves. J. Alloys Compd. 423, 169-171 (2006).

23. Zhan, Y., Bergenti, I., Hueso, L. E. \& Dediu, V. Alignment of energy levels at the $\mathrm{Alq}_{3} / \mathrm{La}_{0.7} \mathrm{Sr}_{0.3} \mathrm{MnO}_{3}$ interface for organic spintronic devices. Phys. Rev. B 76, 045406 (2007).

24. Zhan, Y. Q. et al. Energy level alignment and chemical interaction at Alq3/Co interfaces for organic spintronic devices. Phys. Rev. B 78, 045208 (2008).

25. Zhan, Y. Q. et al. The role of aluminium oxide buffer layer in organic spin-valves performance. Appl. Phys. Lett. 94, 053301 (2009).

26. Tiba, M. V., de Jonge, W. J. M., Koopmans, B. \& Jonkman, H. T. Morphology and electronic properties of the pentacene on cobalt interface. J. Appl. Phys. 100, 093707 (2006).

27. Popinciuc, M., Jonkman, H. T. \& van Wees, B. J. Energy level alignment symmetry at Co/pentacene/Co interfaces. J. Appl. Phys. 100, 093714 (2006).

28. Grobosch, M., Dorr, K., Gangineni, R. B. \& Knupfer, M. Energy level alignment and injection barriers at spin injection contacts between $\mathrm{La}_{0.7} \mathrm{Sr}_{0.3} \mathrm{MnO}_{3}$ and organic semiconductors. Appl. Phys. Lett. 92, 023302 (2008).

29. Lide, D. R. (ed.) Handbook of Physics and Chemistry 90th edn (CRC Press, 2009).

30. Morley, N. A. et al. Room temperature organic spintronics. J. Appl. Phys. 103, 07F306 (2008). 
31. Smith, D. L. \& Ruden, P. P. Spin-polarized tunnelling through potential barriers at ferromagnetic metal/semiconductor Schottky contacts. Phys. Rev. B 78, 125202 (2008).

32. Valenzuela, S. O., Monsma, D. J., Marcus, C. M., Narayanamurti, V. \& Tinkham, M. Spin polarized tunneling at finite bias. Phys. Rev. Lett. 94 196601 (2005).

33. Morenzoni, E. et al. Implantation studies of $\mathrm{keV}$ positive muons in thin metallic layers. Nucl. Instrum. Methods B 192, 254-266 (2002).

34. Prokscha, T. et al. The new $\mu \mathrm{E} 4$ beam at PSI: A hybrid-type large acceptance channel for the generation of a high intensity surface-muon beam. Nucl. Instrum. Methods A 595, 317-331 (2008).

\section{Acknowledgements}

A.J.D. acknowledges financial support from the Royal Society, Leverhulme Trust and the EPSRC (grant EP/G054568/1), C.B. from the SNF (grant 200020-119784 and
200020-129484) and the NCCR program MaNEP and N.A.M. from the British Council. This experimental work was partly carried out at the Swiss Muon Source S $\mu$ S.

\section{Author contributions}

A.J.D. was responsible for project planning and concept. L.S., L.N., M.W., V.K.M., C.B., F.L.P., N.A.M., A.S., G.J.N., T.P., E.M. and A.J.D. were responsible for the experimental measurements. L.S., L.N., W.P.G. and A.J.D. were responsible for the analysis and interpretation of results. P.D., P.S., T.K., W.P.G. and A.J.D. were responsible for the sample growth and characterization.

\section{Additional information}

The authors declare no competing financial interests. Reprints and permissions information is available online at http://npg.nature.com/reprintsandpermissions. Correspondence and requests for materials should be addressed to W.P.G. or A.J.D. 


\section{ERRATUM}

\section{Engineering spin propagation across a hybrid organic/inorganic interface using a}

polar layer

L. Schulz, L. Nuccio, M. Willis, P. Desai, P. Shakya, T. Kreouzis, V. K. Malik, C. Bernhard, F. L. Pratt, N. A. Morley, A. Suter, G. J. Nieuwenhuys, T. Prokscha, E. Morenzoni, W. P. Gillin and A. J. Drew

Nature Materials 10, 39-44 (2011); published online 5 December 2010; corrected after print 18 January 2011.

In the version of this Letter originally published, in the paragraph above the Methods section, 'kiloteslas' should have read ' $k_{\mathrm{B}} T$ '. This has been corrected in the HTML and PDF versions. 